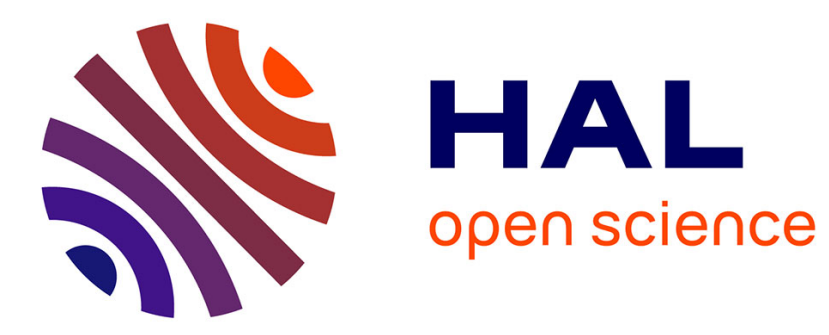

\title{
Cosmic protons
}

J. Alcaraz, B. Alpat, G. Ambrosi, H. Anderhub, L. Ao, A. Arefiev, P.

Azzarello, E. Babucci, Luca Baldini, M. Basile, et al.

\section{To cite this version:}

J. Alcaraz, B. Alpat, G. Ambrosi, H. Anderhub, L. Ao, et al.. Cosmic protons. Physics Letters B, 2000, 490, pp.27-35. 10.1016/S0370-2693(00)00970-9 . in2p3-00006367

HAL Id: in2p3-00006367 https://hal.in2p3.fr/in2p3-00006367

Submitted on 29 Sep 2000

HAL is a multi-disciplinary open access archive for the deposit and dissemination of scientific research documents, whether they are published or not. The documents may come from teaching and research institutions in France or abroad, or from public or private research centers.
L'archive ouverte pluridisciplinaire HAL, est destinée au dépôt et à la diffusion de documents scientifiques de niveau recherche, publiés ou non, émanant des établissements d'enseignement et de recherche français ou étrangers, des laboratoires publics ou privés. 


\section{Cosmic protons}

\section{AMS Collaboration}

J. Alcaraz $^{\text {y }}$, B. Alpat ${ }^{\text {ac }}$, G. Ambrosi ${ }^{\text {r }}$, H. Anderhub ${ }^{\mathrm{ag}}$, L. Ao ${ }^{\mathrm{g}}$, A. Arefiev ${ }^{\mathrm{ab}}$, P. Azzarello ${ }^{\mathrm{r}}$, E. Babucci ${ }^{\text {ac }}$, L. Baldini ${ }^{\mathrm{j}, 1}$, M. Basile ${ }^{\mathrm{j}}$, D. Barancourt ${ }^{\mathrm{s}}$, F. Barao ${ }^{\mathrm{w}, \mathrm{v}}$, G. Barbier ${ }^{\text {s }}$, G. Barreira ${ }^{\text {w }}$, R. Battiston ${ }^{\text {ac }}$, R. Becker ${ }^{1}$, U. Becker ${ }^{1}$, L. Bellagamba ${ }^{j}$, P. Béné ${ }^{\mathrm{r}}$, J. Berdugo ${ }^{\mathrm{y}}$, P. Berges ${ }^{1}$, B. Bertucci ${ }^{\text {ac }}$, A. Biland ${ }^{\mathrm{ag}}, \mathrm{S}$. Bizzaglia ${ }^{\mathrm{ac}}$, S. Blasko ${ }^{\text {ac }}$, G. Boella ${ }^{\mathrm{z}}$, M. Boschini ${ }^{\mathrm{z}}$, M. Bourquin ${ }^{\mathrm{r}}$, L. Brocco ${ }^{\mathrm{j}}$, G. Bruni ${ }^{\mathrm{j}}$, M. Buenerd ${ }^{\text {s }}$, J.D. Burger ${ }^{1}$, W.J. Burger ${ }^{\text {ac }}$, X.D. Cai ${ }^{1}$, C. Camps ${ }^{\mathrm{b}}$, P. Cannarsa ${ }^{\mathrm{ag}}$, M. Capell ${ }^{1}$, D. Casadei ${ }^{\mathrm{j}}$, J. Casaus ${ }^{\mathrm{y}}$, G. Castellini ${ }^{\mathrm{p}}$, C. Cecchi ${ }^{\text {ac }}$, Y.H. Chang ${ }^{\mathrm{m}}$, H.F. Chen ${ }^{\mathrm{t}}$, H.S. Chen ${ }^{\mathrm{i}}$, Z.G. Chen ${ }^{\mathrm{g}}$, N.A. Chernoplekov ${ }^{\text {aa }}$, T.H. Chiueh ${ }^{\mathrm{m}}$, Y.L. Chuang ad , F. Cindolo ${ }^{\mathrm{j}}$, V. Commichau ${ }^{\mathrm{b}}$, A. Contin ${ }^{\mathrm{j}}$, P. Crespo ${ }^{\mathrm{w}}$, M. Cristinziani ${ }^{\mathrm{r}}$, J.P. da Cunha $^{\mathrm{n}}$, T.S. Dai ${ }^{1}$, J.D. Deus ${ }^{\mathrm{v}}$, N. Dinu ${ }^{\mathrm{ac}, 1}$, L. Djambazov ${ }^{\text {ag }}$, I. D’ Antone ${ }^{\mathrm{j}}$, Z.R. Dong ${ }^{\mathrm{h}}$, P. Emonet ${ }^{\mathrm{r}}$, J. Engelberg ${ }^{\text {u }}$, F.J. Eppling ${ }^{1}$, T. Eronen ${ }^{\text {af }}$, G. Esposito ${ }^{\text {ac }}$, P. Extermann ${ }^{\mathrm{r}}$, J. Favier ${ }^{\mathrm{c}}$, E. Fiandrini ${ }^{\mathrm{ac}}$, P.H. Fisher ${ }^{1}$, G. Fluegge ${ }^{\mathrm{b}}$, N. Fouque ${ }^{\mathrm{c}}$, Yu. Galaktionov ${ }^{\mathrm{ab}, \mathrm{l}}$, M. Gervasi ${ }^{\mathrm{z}}$, P. Giusti ${ }^{\mathrm{j}}$, D. Grandi ${ }^{\mathrm{z}}$, O. Grimm ${ }^{\mathrm{ag}}$, W.Q. Gu ${ }^{\mathrm{h}}$, K. Hangarter ${ }^{\mathrm{b}}$, A. Hasan ${ }^{\mathrm{ag}}$, V. Hermel ${ }^{\text {c }}$, H. Hofer ${ }^{\text {ag }}$, M.A. Huang ${ }^{\text {ad }}$, W. Hungerford ${ }^{\text {ag }}$, M. Ionica ${ }^{\text {ac,1, }}$, R. Ionica ${ }^{\text {ac,1 }}$, M. Jongmanns ${ }^{\mathrm{ag}}$, K. Karlamaa ${ }^{\mathrm{u}}$, W. Karpinski ${ }^{\mathrm{a}}$, G. Kenney ${ }^{\mathrm{ag}}$, J. Kenny ${ }^{\text {ac }}$, W. Kim ${ }^{\text {ae }}$, A. Klimentov ${ }^{1, a b}$, R. Kossakowski ${ }^{\text {c }}$, V. Koutsenko ${ }^{1, a b}$, M. Kraeber ${ }^{\text {ag }}$, G. Laborie ${ }^{\text {s }}$, T. Laitinen ${ }^{\text {af }}$, G. Lamanna ${ }^{\text {ac }}$, G. Laurenti ${ }^{\mathrm{j}}$, A. Lebedev ${ }^{1}$, S.C. Lee ${ }^{\text {ad }}$, G. Levi ${ }^{\mathrm{j}}$, P. Levtchenko ${ }^{\text {ac,2 }}{ }^{\text {, C.L. Liu }}{ }^{\mathrm{x}}$,

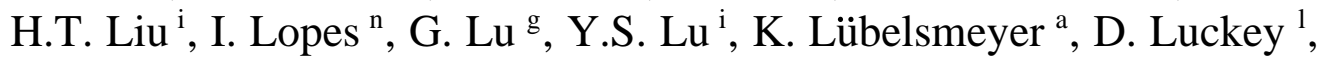
W. Lustermann ${ }^{\mathrm{ag}}$, C. Maña ${ }^{\mathrm{y}}$, A. Margotti ${ }^{\mathrm{j}}$, F. Mayet $^{\mathrm{s}}$, R.R. McNeil ${ }^{\mathrm{d}}$, B. Meillon ${ }^{\mathrm{s}}$, M. Menichelli ${ }^{\text {ac }}$, A. Mihul ${ }^{\mathrm{k}}$, A. Mourao ${ }^{\mathrm{v}}$, A. Mujunen ${ }^{\mathrm{u}}$, F. Palmonari ${ }^{\text {j }}$, A. Papi ${ }^{\text {ac }}$, I.H. Park ${ }^{\text {ae }}$, M. Pauluzzi ${ }^{\text {ac }}$, F. Pauss ${ }^{\text {ag }}$, E. Perrin ${ }^{\mathrm{r}}$, A. Pesci ${ }^{\mathrm{j}}$, A. Pevsner ${ }^{\mathrm{e}}$, M. Pimenta ${ }^{\mathrm{w}, \mathrm{v}}$, V. Plyaskin ${ }^{\mathrm{ab}}$, V. Pojidaev ${ }^{\mathrm{ab}}$, 
M. Pohl ${ }^{\mathrm{r}}$, V. Postolache ${ }^{\text {ac, }, \text { N N. Produit }}{ }^{\mathrm{r}}$, P.G. Rancoita ${ }^{\mathrm{z}}$, D. Rapin ${ }^{\mathrm{r}}$, F. Raupach ${ }^{\text {a }}$, D. Ren ${ }^{\text {ag }}$, Z. Ren ${ }^{\text {ad }}$, M. Ribordy ${ }^{\mathrm{r}}$, J.P. Richeux ${ }^{\mathrm{r}}$, E. Riihonen ${ }^{\text {af }}$, J. Ritakari ${ }^{\text {u }}$, U. Roeser ${ }^{\text {ag }}$, C. Roissin ${ }^{\text {s, R. Sagdeev }}{ }^{\circ}$, G. Sartorelli ${ }^{j}$, A. Schultz von Dratzig a , G. Schwering ${ }^{\text {a }}$, G. Scolieri ${ }^{\text {ac }}$, E.S. Seo ${ }^{\circ}$, V. Shoutko ${ }^{1}$, E. Shoumilov ${ }^{\text {ab }}$, R. Siedling ${ }^{\text {a }}$, D. Son ${ }^{\text {ae }}$, T. Song ${ }^{\text {h }}$, M. Steuer ${ }^{1}$, G.S. Sun ${ }^{\text {h}}$, H. Suter ${ }^{\mathrm{ag}}$, X.W. Tang ${ }^{\mathrm{i}}$, Samuel C.C. Ting ${ }^{1}$, S.M. Ting ${ }^{1}$, M. Tornikoski ${ }^{\text {, }}$, J. Torsti ${ }^{\text {af }}$, J. Trümper ${ }^{\text {q }}$, J. Ulbricht ${ }^{\text {ag }}$, S. Urpo ${ }^{\text {u }}$, I. Usoskin ${ }^{\mathrm{z}}$, E. Valtonen ${ }^{\text {af }}$, J. Vandenhirtz ${ }^{\text {a }}$, F. Velcea ${ }^{\text {ac, }, 1}$, E. Velikhov ${ }^{\text {aa }}$, B. Verlaat ${ }^{\text {ag,3 }}{ }^{\text {, I. Vetlitsky }}{ }^{\text {ab }}$, F. Vezzu ${ }^{\text {s }}$, J.P. Vialle ${ }^{\mathrm{c}}$, G. Viertel ${ }^{\mathrm{ag}}$, D. Vité ${ }^{\mathrm{r}}$, H. Von Gunten ${ }^{\mathrm{ag}}$, S. Waldmeier Wicki ${ }^{\text {ag }}$, W. Wallraff ${ }^{\text {a }}$, B.C. Wang ${ }^{\text {x }}$, J.Z. Wang ${ }^{\text {g }}$, Y.H. Wang ${ }^{\text {ad }}$, K. Wiik ${ }^{\mathrm{u}}$, C. Williams ${ }^{\mathrm{j}}$, S.X. Wu ${ }^{1, \mathrm{~m}}$, P.C. Xia ${ }^{\mathrm{h}}$, J.L. Yan ${ }^{\mathrm{g}}$, L.G. Yan ${ }^{\mathrm{h}}$, C.G. Yang ${ }^{\mathrm{i}}$, M. Yang ${ }^{\text {i, }}$ S.W. Ye ${ }^{\mathrm{t}, 4}$, P. Yeh ${ }^{\text {ad }}$, Z.Z. Xu ${ }^{\mathrm{t}}$, H.Y. Zhang ${ }^{\mathrm{f}}$, Z.P. Zhang ${ }^{\text {t }}$ D.X. Zhao ${ }^{\text {h }}$, G.Y. Zhu ${ }^{\mathrm{i}}$, W.Z. Zhu ${ }^{\mathrm{g}}$, H.L. Zhuang ${ }^{\mathrm{i}}$, A. Zichichi ${ }^{\mathrm{j}}$, B. Zimmermann ${ }^{\text {ag }}$

\footnotetext{
${ }^{a}$ I. Physikalisches Institut, RWTH, D-52056 Aachen, Germany ${ }^{5}$

${ }^{\text {b III. Physikalisches Institut, RWTH, D-52056 Aachen, Germany }}{ }^{5}$

c Laboratoire d'Annecy-le-Vieux de Physique des Particules, LAPP, F-74941 Annecy-le-Vieux CEDEX, France

${ }^{\mathrm{d}}$ Louisiana State University, Baton Rouge, LA 70803, USA

e Johns Hopkins University, Baltimore, MD 21218, USA

${ }^{\mathrm{f}}$ Center of Space Science and Application, Chinese Academy of Sciences, 100080 Beijing, China

${ }^{\mathrm{g}}$ Chinese Academy of Launching Vehicle Technology, CALT, 100076 Beijing, China

${ }^{\mathrm{h}}$ Institute of Electrical Engineering, IEE, Chinese Academy of Sciences, 100080 Beijing, China

${ }^{\mathrm{i}}$ Institute of High Energy Physics, IHEP, Chinese Academy of Sciences, 100039 Beijing, China ${ }^{6}$

${ }^{\mathrm{j}}$ University of Bologna and INFN-Sezione di Bologna, I-40126 Bologna, Italy

${ }^{\mathrm{k}}$ Institute of Microtechnology, Politechnica University of Bucharest and University of Bucharest, R-76900 Bucharest, Romania

${ }^{1}$ Massachusetts Institute of Technology, Cambridge, MA 02139, USA

${ }^{\mathrm{m}}$ National Central University, Chung-Li 32054, Taiwan, ROC

${ }^{\mathrm{n}}$ Laboratorio de Instrumentacao e Fisica Experimental de Particulas, LIP, P-3000 Coimbra, Portugal

${ }^{\circ}$ University of Maryland, College Park, MD 20742, USA

${ }^{\mathrm{p}}$ INFN Sezione di Firenze, I-50125 Florence, Italy

${ }^{\mathrm{q}}$ Max-Plank Institut fur Extraterrestrische Physik, D-85740 Garching, Germany

${ }^{\mathrm{r}}$ University of Geneva, CH-1211 Geneva 4, Switzerland

${ }^{\mathrm{s}}$ Institut des Sciences Nucleaires, F-38026 Grenoble, France

${ }^{t}$ Chinese University of Science and Technology, USTC, Hefei, Anhui 230 029, China ${ }^{6}$

" Helsinki University of Technology, FIN-02540 Kylmala, Finland

${ }^{v}$ Instituto Superior Técnico, IST, P-1096 Lisboa, Portugal

${ }^{\mathrm{w}}$ Laboratorio de Instrumentacao e Fisica Experimental de Particulas, LIP, P-1000 Lisboa, Portugal

${ }^{\mathrm{x}}$ Chung-Shan Institute of Science and Technology, Lung-Tan, Tao Yuan 325, Taiwan, ROC

${ }^{\text {y } C e n t r o ~ d e ~ I n v e s t i g a c i o n e s ~ E n e r g e ́ t i c a s, ~ M e d i o a m b i e n t a l e s ~ y ~ T e c n o l o g i ́ c a s, ~ C I E M A T, ~ E-28040 ~ M a d r i d, ~ S p a i n ~}{ }^{7}$

${ }^{\mathrm{z}}$ INFN-Sezione di Milano, I-20133 Milan, Italy

${ }^{\text {aa }}$ Kurchatov Institute, Moscow, 123182, Russia

${ }^{a b}$ Institute of Theoretical and Experimental Physics, ITEP, Moscow, 117259, Russia

${ }^{\text {ac }}$ INFN-Sezione di Perugia and Universitá Degli Studi di Perugia, I-06100 Perugia, Italy ${ }^{8}$

ad Academia Sinica, Taipei 11529, Taiwan, ROC

ae Kyungpook National University, 702-701 Taegu, South Korea

af University of Turku, FIN-20014 Turku, Finland

ag Eidgenössische Technische Hochschule, ETH Zürich, CH-8093 Zürich, Switzerland
}

Received 26 July 2000; accepted 11 August 2000

Editor: K. Winter 


\section{Abstract}

The primary proton spectrum in the kinetic energy range 0.2 to $200 \mathrm{GeV}$ was measured by the Alpha Magnetic Spectrometer (AMS) during space shuttle flight STS-91 at an altitude of $380 \mathrm{~km}$. The complete data set combining three shuttle attitudes and including all known systematic effects is presented. (C) 2000 Elsevier Science B.V. All rights reserved.

\section{Introduction}

Accurate measurements of primary cosmic ray spectra, particularly of protons, are important for atmospheric neutrinos studies and studies of neutrino oscillation phenomenon [1,2].

The Alpha Magnetic Spectrometer (AMS) [3] is a high energy physics experiment scheduled for installation on the International Space Station. In preparation for this long duration mission, AMS flew a precursor mission on board of the space shuttle Discovery during flight STS-91 in June 1998. The high statistic measurements of the primary proton spectra by AMS, free from atmospheric corrections, provide reliable information for atmospheric neutrino flux calculations.

In a previous paper [4] we presented the general features of protons in near Earth orbit. Measuring the distribution as a function of energy and latitude enabled us to study the behaviour of the proton flux near Earth. Above the geomagnetic cutoff the observed spectrum can be parameterized by a power law [5]. Below the cutoff a substantial second spectrum concentrated at equatorial latitudes with a flux of $\sim 70 \mathrm{~m}^{-2} \mathrm{~s}^{-1} \mathrm{sr}^{-1}$ is observed. In near Earth orbit, between the atmosphere and the radiation belts,

\footnotetext{
${ }^{1}$ Permanent address: HEPPG, Univ. of Bucharest, Romania.

${ }^{2}$ Permanent address: Nuclear Physics Institute, St. Petersburg, Russia.

${ }^{3}$ Now at National Institute for High Energy Physics, NIKHEF, NL-1009 DB Amsterdam, The Netherlands.

${ }^{4}$ Supported by ETH Zürich.

${ }^{5}$ Supported by the Deutsches Zentrum für Luft- und Raumfahrt, DLR.

${ }^{6}$ Supported by the National Natural Science Foundation of China.

${ }^{7}$ Also supported by the Comisión Interministerial de Ciencia y Tecnología.

${ }^{8}$ Also supported by the Italian Space Agency.
}

these second spectrum particles follow a complicated path in the Earth's magnetic field. This behaviour is different from that extrapolated from satellite observations in the radiation belts. It is also different from that extrapolated from balloon observations in the upper layer of the atmosphere. A striking feature of the second spectrum is that most of the protons originate from a very restricted geographic region.

In this paper all the available events are used to determine the primary proton spectrum. This increase in statistics enables us to present an accurate determination of the primary proton flux.

\section{The AMS experiment on STS-91}

The major elements of AMS as flown on STS-91 consisted of a permanent magnet, a six plane tracker, time of flight hodoscopes, a Cerenkov counter and anticoincidence counters. The AMS detector has been described elsewhere $[4,6]$.

For this study, events were collected in three periods during which the shuttle attitude was constrained, within $1^{\circ}$, to keep the AMS positive z-axis pointing within $0^{\circ}, 20^{\circ}$ and $45^{\circ}$ of the zenith. The acceptance was restricted to events with an incident angle, $\theta$, within $38^{\circ}$ of the positive z-axis of AMS. The orbital inclination was $51.7^{\circ}$ and the geodetic altitude during these periods ranged from 350 to 390 $\mathrm{km}$. Data taken while orbiting in or near the South Atlantic Anomaly were excluded.

The response of the detector was simulated using the AMS detector simulation program, based on the GEANT package [7]. The effects of energy loss, multiple scattering, interactions, decays and the measured detector efficiency and resolution were included.

After the flight, the AMS detector was extensively calibrated at two accelerators: at GSI, Darm- 
stadt, with helium and carbon beams at 600 incident angles and locations and $10^{7}$ events, and at the CERN proton-synchrotron machine in the energy region of 2 to $14 \mathrm{GeV}$, with 1200 incident angles and locations and $10^{8}$ events. This ensured that the performance of the detector and the analysis procedure were thoroughly understood.

\section{Analysis}

Proton events were reconstructed and selected as described in our earlier paper [4]. Then events with rigidity, $R=p c /|Z| e$, well above the geomagnetic cutoff were selected:

$R>R_{C} \times\left(1.2+2 \sigma\left(R_{C}\right)\right)$

where $R_{C}$ was the maximum cutoff rigidity and $\sigma\left(R_{C}\right)$ was the measurement uncertainty at that rigidity. $R_{C}$ was calculated in the dipole approximation for one second time intervals along the shuttle trajectory for the measured shuttle attitude. As protons are the dominant components in cosmic rays, after selecting events with $Z=+1$, the proton sample has only minor backgrounds which consist of charged pions and deuterons. The estimated fraction of charged pions, which are produced in the top part of AMS, with energy below $0.5 \mathrm{GeV}$ is $1 \%$. Above this energy the fraction decreases rapidly with increasing energy. The deuteron abundance in cosmic rays above the geomagnetic cutoff is about $2 \%$. To remove low energy charged pions and deuterons, the measured mass was required to be within 3 standard deviations of the proton mass. This rejected about $3 \%$ of the events while reducing the background contamination to negligible levels over all energies.

The proton fluxes were derived from the measured counting rates using the acceptance as a function of the proton momentum. Protons with different momenta and directions were generated via the Monte Carlo method, passed through the AMS detector simulation program and accepted if the trigger and reconstruction requirements were satisfied as for the data. The acceptance was found to be $0.15 \mathrm{~m}^{2} \mathrm{sr}$ on average and only weakly momentum dependent. The

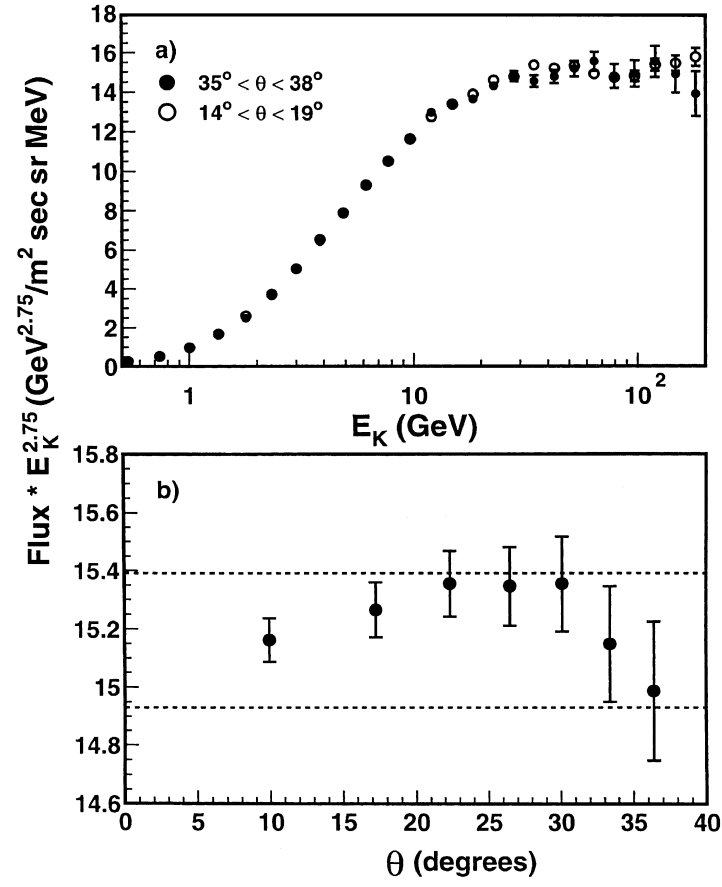

Fig. 1. Isotropy: (a) Comparison of the energy spectra for two angular acceptance ranges. (b) Fluxes averaged above $20 \mathrm{GeV}$ as a function of the incident particle angle. Error bars show statistical errors. The dashed lines in (b) indicate the estimated range of systematic errors for this case. As seen, the data are independent of $\theta$.

acceptance was then corrected following an analysis of unbiased trigger events ${ }^{9}$ [4].

The observed primary proton spectrum was verified to be isotropic as seen in Fig. 1a where the spectra in kinetic energy, $E_{\mathrm{K}}$, scaled by $E_{\mathrm{K}}^{2.75}$ are compared for two intervals of incident angle to the detector, $\theta$, and in Fig. 1b, where the average fluxes are shown as functions of the incident angle. Both are in agreement with an isotropic distribution. Therefore the three data collection periods are combined to obtain a data set having a total of 5.6 million primary protons. Given the resulting small statistical errors, further detailed studies of the systematic effects were performed.

\footnotetext{
${ }^{9}$ This analysis removes possible systematics of the Monte Carlo method [7].
} 
Table 1

Average systematics of the trigger and reconstruction, $\varepsilon_{\text {sys } 1}$

\begin{tabular}{ll}
\hline Source & Error $(\%)$ \\
\hline fast trigger & 1.5 \\
anti trigger & 1 \\
Level3 TOF & 2 \\
Level3 tracker & 1.5 \\
track and velocity fit & 1.5 \\
total & 3.5 \\
\hline
\end{tabular}

The first source of systematic error, $\varepsilon_{\text {sys } 1}$, was due to trigger efficiency variations and event reconstruction accuracy variations, both of which are related to the background rates and detector temperatures at different times and at different shuttle locations and orientations and to the energy deposited by the proton in each detector layer. Above $\sim 20 \mathrm{GeV}$ this systematic error is nearly energy independent. The calculated average contributions are shown in Table 1 , the total error from this origin is $3.5 \%$.

A second source of systematic effects, $\varepsilon_{\text {sys } 2}$, arose from Monte Carlo corrections. These errors are energy dependent but uncorrelated between energy bins. The calculated average contributions are shown in Table 2 and total to $3 \%$. The contributions of $\varepsilon_{\text {sys } 1}$ and $\varepsilon_{\text {sys } 2}$ versus energy are detailed in Table 3 .

A third source of systematics, $\varepsilon_{\text {sys } 3}$, is from the unfolding used to obtain the incident differential spectrum from the measured spectrum based on resolution functions obtained by simulation. These errors are bin to bin correlated. As detailed in Table 3, they are typically $1 \%$ below $\sim 20 \mathrm{GeV}$ and reach $5 \%$ at $\sim 100 \mathrm{GeV}$.

Table 2

Systematics of the Monte Carlo corrections leading to the energy dependent $\varepsilon_{\text {sys } 2}$

\begin{tabular}{ll}
\hline Source & Error $(\%)$ \\
\hline particle interactions & 1.5 \\
Monte Carlo statistics & 1.5 \\
proton selection & 2 \\
geomagnetic cutoff & 0.5 \\
total & 3.0 \\
\hline
\end{tabular}

Table 3

Primary proton spectrum. Data collected during the three periods with different zenith pointing criteria are combined. Kinetic energy is in $\mathrm{GeV}$, flux in $\left(\mathrm{m}^{2} \mathrm{srs} \mathrm{MeV}\right)^{-1}, \varepsilon_{\text {stat }}$ stands for the statistical error and $\varepsilon_{\mathrm{sys} 1,2,3}$ for the systematic errors

\begin{tabular}{ll}
\hline Kinetic energy & Flux $\pm \varepsilon_{\text {stat }} \pm \varepsilon_{\text {sys } 1} \pm \varepsilon_{\text {sys } 2} \pm \varepsilon_{\text {sys } 3}$ \\
\hline $0.22-0.31$ & $(154 . \pm 1.6 \pm 5.9 \pm 4.0 \pm 1.9) \times 10^{-2}$ \\
$0.31-0.44$ & $(156 . \pm .99 \pm 6.0 \pm 3.8 \pm 1.3) \times 10^{-2}$ \\
$0.44-0.62$ & $(143 . \pm .59 \pm 6.0 \pm 3.6 \pm 1.0) \times 10^{-2}$ \\
$0.62-0.85$ & $(120 . \pm .39 \pm 4.6 \pm 3.1 \pm .82) \times 10^{-2}$ \\
$0.85-1.15$ & $(966 . \pm 2.6 \pm 37 . \pm 24 . \pm 6.7) \times 10^{-3}$ \\
$1.15-1.54$ & $(738 . \pm 1.8 \pm 28 . \pm 18 . \pm 5.1) \times 10^{-3}$ \\
$1.54-2.02$ & $(533 . \pm 1.2 \pm 20 . \pm 13 . \pm 3.4) \times 10^{-3}$ \\
$2.02-2.62$ & $(372 . \pm .80 \pm 14 . \pm 8.9 \pm 2.7) \times 10^{-3}$ \\
$2.62-3.38$ & $(247 . \pm .53 \pm 9.5 \pm 5.8 \pm 1.8) \times 10^{-3}$ \\
$3.38-4.31$ & $(161 . \pm .33 \pm 6.2 \pm 3.7 \pm 1.3) \times 10^{-3}$ \\
$4.31-5.45$ & $(101 . \pm .20 \pm 3.9 \pm 2.3 \pm .74) \times 10^{-3}$ \\
$5.45-6.86$ & $(630 . \pm 1.3 \pm 24 . \pm 14 . \pm 5.2) \times 10^{-4}$ \\
$6.86-8.60$ & $(378 . \pm .84 \pm 14 . \pm 8.6 \pm 3.3) \times 10^{-4}$ \\
$8.60-10.7$ & $(226 . \pm .54 \pm 8.7 \pm 5.2 \pm 2.0) \times 10^{-4}$ \\
$10.7-13.3$ & $(135 . \pm .36 \pm 5.2 \pm 3.1 \pm 1.5) \times 10^{-4}$ \\
$13.3-16.5$ & $(786 . \pm 2.3 \pm 30 . \pm 18 . \pm 10.) \times 10^{-5}$ \\
$16.5-20.5$ & $(449 . \pm 1.5 \pm 17 . \pm 11 . \pm 6.6) \times 10^{-5}$ \\
$20.5-25.3$ & $(266 . \pm .98 \pm 10 . \pm 6.4 \pm 4.3) \times 10^{-5}$ \\
$25.3-31.2$ & $(148 . \pm .61 \pm 5.7 \pm 3.7 \pm 2.7) \times 10^{-5}$ \\
$31.2-38.4$ & $(856 . \pm 4.0 \pm 33 . \pm 22 . \pm 16.) \times 10^{-6}$ \\
$38.4-47.3$ & $(496 . \pm 2.7 \pm 19 . \pm 13 . \pm 9.2) \times 10^{-6}$ \\
$47.3-58.2$ & $(284 . \pm 1.8 \pm 11 . \pm 7.9 \pm 5.7) \times 10^{-6}$ \\
$58.2-71.5$ & $(154 . \pm 1.2 \pm 5.9 \pm 4.4 \pm 3.0) \times 10^{-6}$ \\
$71.5-87.8$ & $(86.2 \pm .80 \pm 3.3 \pm 2.4 \pm 1.7) \times 10^{-6}$ \\
$87.8-108$. & $(49.4 \pm .55 \pm 1.9 \pm 1.3 \pm .94) \times 10^{-6}$ \\
$108 .-132$. & $(29.0 \pm .40 \pm 1.1 \pm .78 \pm 1.1) \times 10^{-6}$ \\
$132 .-162$. & $(16.4 \pm .27 \pm .63 \pm .44 \pm .80) \times 10^{-6}$ \\
$162 .-199$. & $(9.39 \pm .18 \pm .36 \pm .25 \pm 1.0) \times 10^{-6}$ \\
\hline &
\end{tabular}

Fig. 2 shows the primary proton spectrum with the statistical and three systematic errors combined in quadrature.

\section{Verification of the systematic errors}

A careful experimental verification of the calculated systematic errors was performed. The calculated error took into account the propagation of the statistical and three systematic errors. We present 6 examples:

1. Fig. 3 shows the flux variation, $\delta \equiv$ (flux /average flux) -1 as a function of energy, for the three 


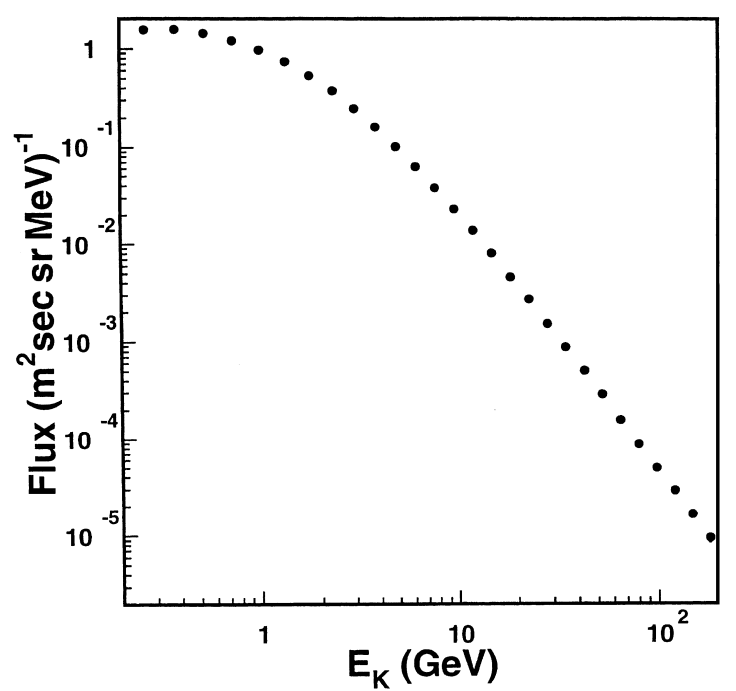

Fig. 2. The primary proton flux. Fitting this spectrum to a power law in rigidity, $\Phi=\Phi_{0} R^{-\gamma}$, over the range $10 \mathrm{GeV}<R<200$ $\mathrm{GeV}$ yields $\gamma=2.78 \pm 0.009$ (fit) \pm 0.019 (sys) and $\Phi_{0}=17.1 \pm$ 0.15 (fit) \pm 1.3 (sys) $\pm 1.5(\gamma) \mathrm{GeV}^{2.78} /\left(\mathrm{m}^{2} \mathrm{~s} \mathrm{sr} \mathrm{MeV}\right)$, in good agreement with our previous result [4].

data collection periods, corresponding to the three different shuttle orientations, compared with the systematic error calculated for this case.

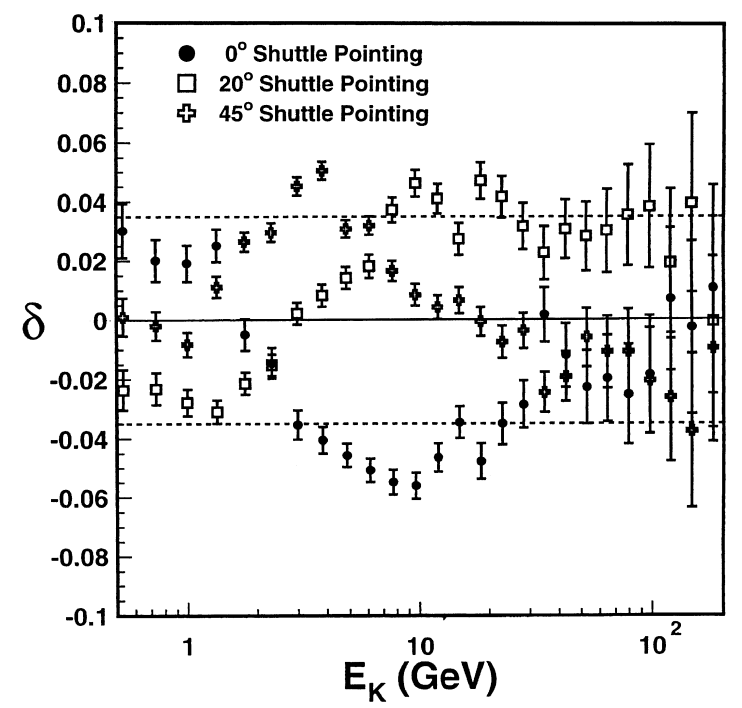

Fig. 3. Systematic error verification: Flux variation, $\delta$, as a function of proton energy for different shuttle orientations. Errors shown are statistical. The dashed lines show the range of systematic error calculated for this case.

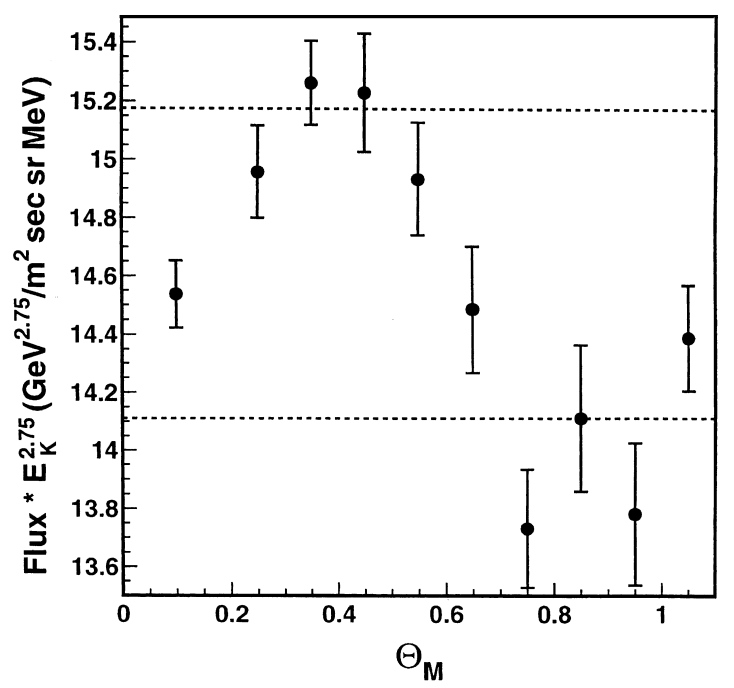

Fig. 4. Systematic error verification: Fluxes averaged above 20 $\mathrm{GeV}$ scaled by $E_{\mathrm{K}}^{2.75}$ as a function of magnetic latitude. Errors shown are statistical. The dashed lines show the range of the systematic error calculated for this case.

2. Fig. 4 shows the flux averaged above $20 \mathrm{GeV}$ versus different intervals of the corrected geomagnetic latitude [8], $\Theta_{\mathrm{M}}$, at which the protons were detected compared with the systematic error calculated for this case.

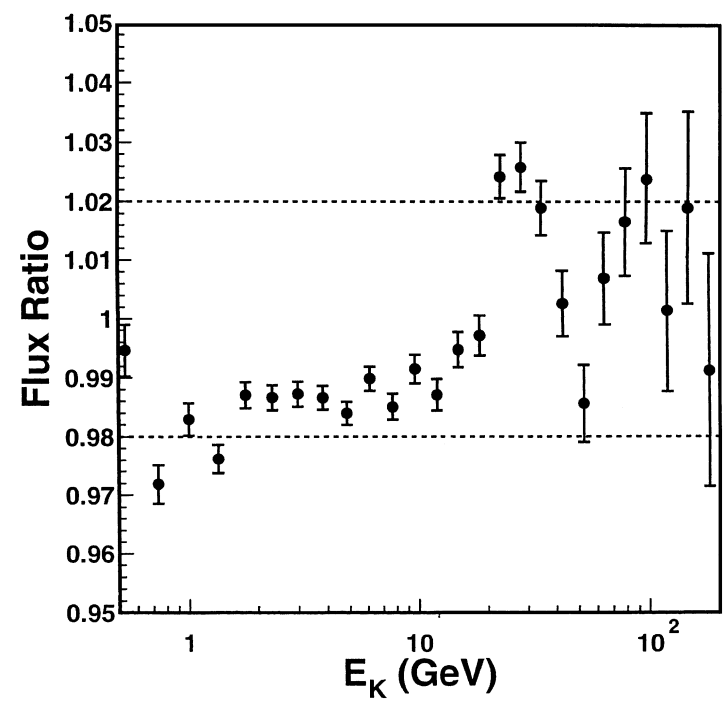

Fig. 5. Verification of systematics: Ratio of the fluxes computed with and without proton selection quality cuts. The dashed lines show the range of the average systematic error calculated for this case. 


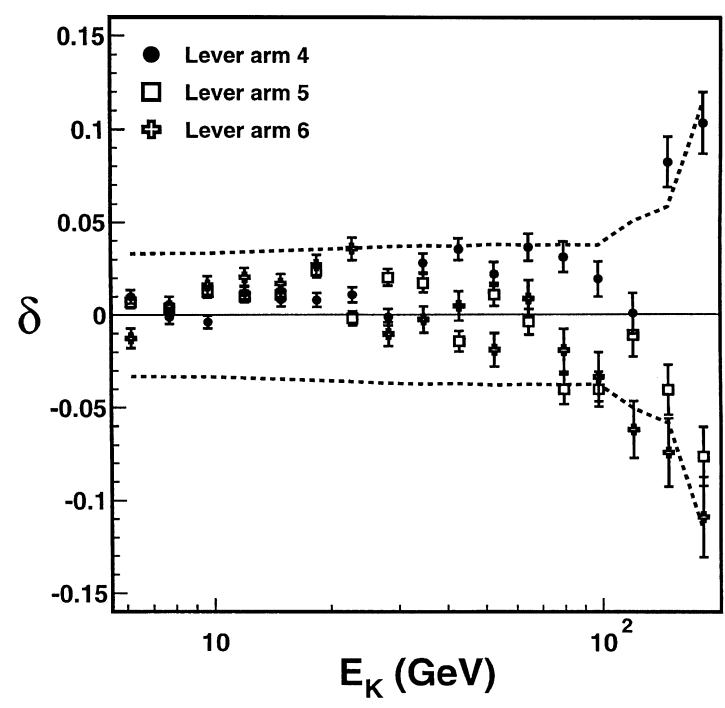

Fig. 6. Verification of systematics: Flux variation, $\delta$, for events with lever arms in the tracker which correspond to 4,5 or 6 planes. The dashed lines show the range of the calculated systematic errors.
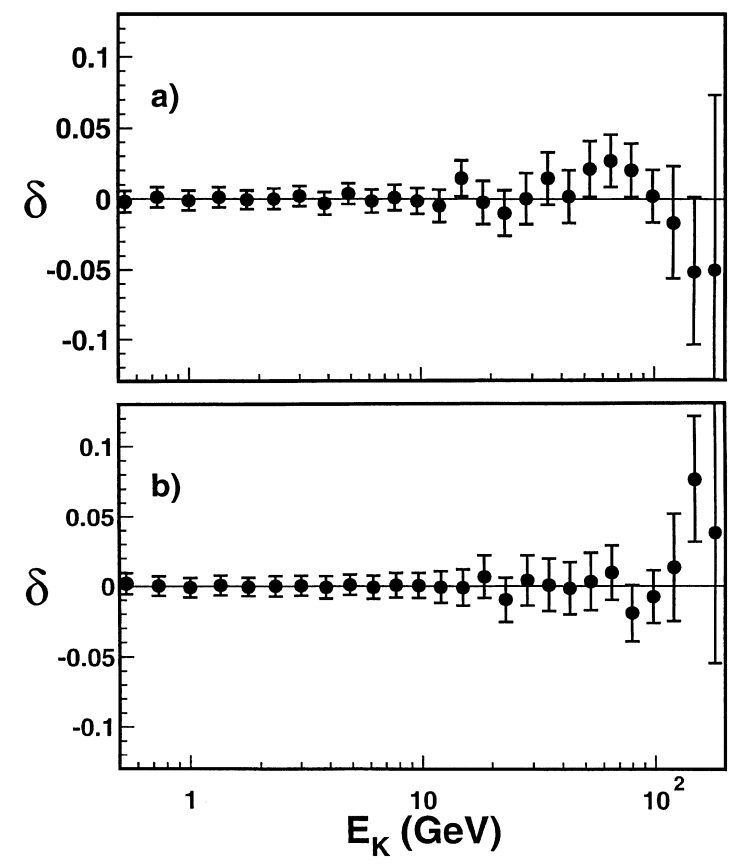

Fig. 7. Verification of systematics: (a) Variation of the flux, $\delta$, computed with a resolution matrix including an artificial smearing of the alignment by an additional $10 \mu \mathrm{m}$. (b) Variation of the flux, $\delta$, calculated with two different unfolding methods.
3. Fig. 5 shows the ratio of the flux with and without proton selection quality cuts. As seen, the variation is within the systematic error for this case.

4. Fig. 6 shows the variation of the flux, $\delta$, for events which had different lever arms in the tracker, corresponding to the number of tracker planes used in the fit. Again, the variation is within the range of the systematic errors for this case.

5. The systematic error assigned to the unfolding was checked at several energies using the test beam measurements. Fig. 7a shows how the spectrum changes if the unfolding matrix is artificially varied by smearing the alignment between different tracker ladders by an additional $10 \mu \mathrm{m}$. At $\sim 100 \mathrm{GeV}$ this corresponds to varying the momentum resolution by $15 \%$.

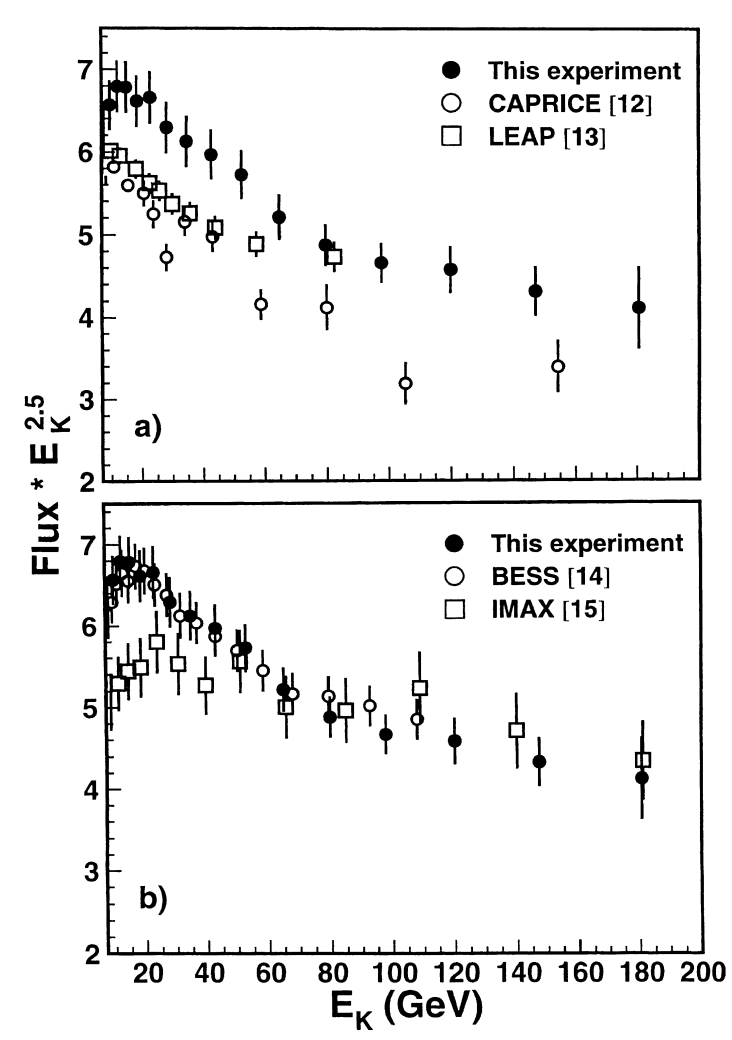

Fig. 8. The primary proton spectrum multiplied by $E_{\mathrm{K}}^{2.5}$ in units of $\mathrm{GeV}^{2.5} /\left(\mathrm{m}^{2} \mathrm{~s} \mathrm{srMeV}\right)$ as measured by this experiment (total errors shown) in comparison with some recent balloon based measurements. 
6. Fig. $7 \mathrm{~b}$ gives an estimate of the accuracy of the unfolding method by comparing two completely different deconvolution techniques [9-11]:

6.1. The solution of an overdetermined system of linear equations by a method of converged weights [4].

6.2. 'Classical' unfolding by regularization, namely minimization of the function [11]:

$$
\begin{aligned}
& \left(\frac{\int f\left(x^{\prime}\right) K\left(x, x^{\prime}\right) d x^{\prime}-g(x)}{\varepsilon(x)}\right)^{2} \\
& +\alpha\left(\frac{d^{2} f}{d x^{2}}\right)^{2}
\end{aligned}
$$

where $g(x)$ is the measurement, $\varepsilon(x)$ its error, $K\left(k, x^{\prime}\right)$ is the resolution matrix, $f(x)$ is the solution and $\alpha$ is a regularization parameter. This method tends to give a smoother solution, since the searched function is a priori assumed to be a 'smooth' one, with the most probable 'smoothness' estimated from Bayes' theorem.

$\mathrm{Up}$ to $\sim 100 \mathrm{GeV}$ the two methods agree within 2-3\%. As the unfolding systematics were understood for both methods, the average of the two procedures was used. The input errors for both procedures were the corresponding statistical errors and the systematics of the resolution matrix used ${ }^{10}$.

In these examples, and in all other cases, the assigned systematic errors were found to be correct.

\section{Results}

The primary proton spectrum together with the statistical and three systematic errors are presented in Table 3. For comparison with balloon measurements [12-15] the data has been scaled by $E_{\mathrm{K}}^{2.5}$ as shown in

\footnotetext{
${ }^{10}$ In our previous publication [4] the flux dependence on energy and latitude was presented. These fluxes cannot be combined as such since the errors are correlated through the systematics of the resolution matrix.
}

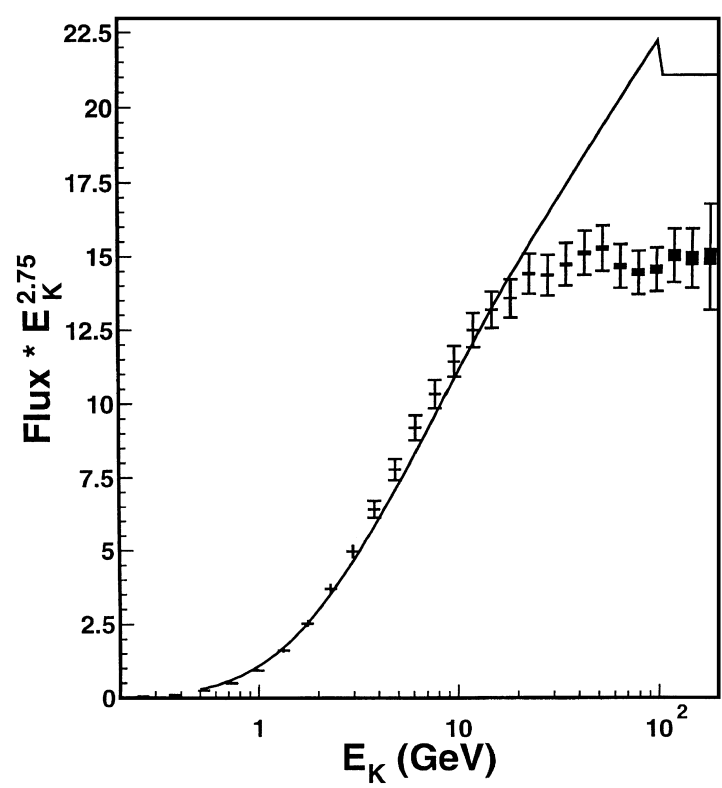

Fig. 9. The primary proton flux multiplied by $E_{\mathrm{K}}^{2.75}$ in units of $\mathrm{GeV}^{2.75} /\left(\mathrm{m}^{2} \mathrm{~s} \mathrm{srMeV}\right)$. The solid rectangles indicate the statistical errors, the error bars indicate the total error. The solid line is the flux used to calculate atmospheric neutrinos [2] scaled by $E_{\mathrm{K}}^{2.75}$.

Fig. 8. The flux scaled by $E_{\mathrm{K}}^{2.75}$ is shown in Fig. 9 . As seen from Fig. 9, our data is a smooth function which is flat above $20 \mathrm{GeV}$. This is a substantial deviation from the current estimates used to calculate atmospheric neutrinos [2].

\section{Acknowledgements}

The support of INFN, Italy, ETH-Zürich, the University of Geneva, the Chinese Academy of Sciences, Academia Sinica and National Central University, Taiwan, the RWTH-Aachen, Germany, the University of Turku, the University of Technology of Helsinki, Finland, the US DOE and MIT, CIEMAT, Spain, LIP, Portugal and IN2P3, France, is gratefully acknowledged.

\section{References}

[1] Y. Fukuda et al., Phys. Lett. B 436 (1998) 33.

[2] M. Honda et al., Phys. Rev. D 52 (1995) 4985; G. Battistoni 
et al., hep-ph/9907408; Y. Tserkovnyak et al., hep-ph/ 9907450; P. Lipari, hep-ph/9905506; T. Gaisser, hep-ph/ 0001027 ; N. Fornengo et al., hep-ph/0002147.

[3] S. Ahlen et al., Nucl. Inst. Meth. A 350 (1994) 351.

[4] AMS Collaboration, J. Alcaraz et al., Phys. Lett. B 472 (2000) 215.

[5] A.D. Erlykin et al., Phys. Lett. B 482 (2000) 337.

[6] AMS Collaboration, J. Alcaraz et al., Phys. Lett. B 461 (1999) 387.

[7] R. Brun et al., GEANT 3, CERN DD/EE/84-1, Revised, 1987; P.A. Aamio et al., FLUKA Users Guide, CERN TIS-RP-190, 1990.

[8] A. Brekke, Physics of the Upper Polar Atmosphere, Wiley, New York, 1997, pp. 127-145.
[9] A. Kondor, Nucl. Inst. Meth. 216 (1983) 177; G. D'Agostini, Nucl. Inst. Meth. A 362 (1995) 487.

[10] V. Blobel, Unfolding Methods in High Energy Physics, Proc. 1984 CERN School of Computing, CERN 85-09 (1985) 88.

[11] V.F. Turchin, V.A. Nozik, Izv. Acad. Nauk USSR ser. Fiz 5 (1969) 29; V.F. Turchin, V.P. Kozlov, M.S. Malkevich, Phys. Usp. 102 (1970) 345.

[12] M. Boesio et al., ApJ 518 (1999) 457.

[13] E.S. Seo et al., ApJ 378 (1991) 763.

[14] T. Sanuki et al., astro-ph/0002481.

[15] W. Menn et al., ApJ 533 (2000) 281. 